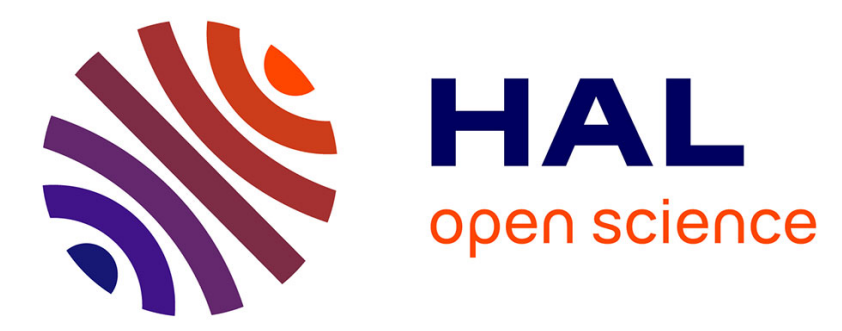

\title{
Electro-click construction of hybrid nanocapsule films with triggered delivery properties
}

Flavien Sciortino, Gaulthier Rydzek, Fabien Grasset, Myrtil L Kahn, Jonathan P Hill, Soizic Chevance, Fabienne Gauffre, Katsuhiko Ariga

\section{To cite this version:}

Flavien Sciortino, Gaulthier Rydzek, Fabien Grasset, Myrtil L Kahn, Jonathan P Hill, et al.. Electroclick construction of hybrid nanocapsule films with triggered delivery properties. Physical Chemistry Chemical Physics, 2018, 20 (4), pp.2761-2770. 10.1039/c7cp07506e . hal-01684549

\author{
HAL Id: hal-01684549 \\ https://hal.science/hal-01684549
}

Submitted on 11 Sep 2020

HAL is a multi-disciplinary open access archive for the deposit and dissemination of scientific research documents, whether they are published or not. The documents may come from teaching and research institutions in France or abroad, or from public or private research centers.
L'archive ouverte pluridisciplinaire HAL, est destinée au dépôt et à la diffusion de documents scientifiques de niveau recherche, publiés ou non, émanant des établissements d'enseignement et de recherche français ou étrangers, des laboratoires publics ou privés. 


\title{
PCCP
}

ARTICLE

\section{Electro-click construction of Hybrid Nanocapsule Films with Triggered Delivery Properties}

Received 00th January 20xx, Accepted 00th January 20xx

DOI: $10.1039 / x 0 x \times 00000 x$

www.rsc.org/
Flavien Sciortino ${ }^{\dagger a} *$, Gaulthier Rydzek ${ }^{\dagger b} *$, Fabien Grasset ${ }^{c}$, Myrtil L. Kahn ${ }^{d}$, Jonathan P. Hill ${ }^{b}$, Soizic Chevance $^{a}$, Fabienne Gauffre ${ }^{\text {, Katsuhiko Ariga }}{ }^{\text {,e }}$

\begin{abstract}
Hollow nanocapsules (named Hybridosomes ${ }^{\circledR}$ ) possessing a polymer/nanoparticles shell were used to covalently construct hybrid films in a one-pot fashion. Alkyne bearing organic/inorganic Hybridosomes ${ }^{\circledR}$ were reticulated with azide bearing homobifunctional polyethyleneglycol (PEG) linkers, by using an electro-click reaction on $\mathrm{F}_{-} \mathrm{SnO}_{2}$ (FTO) electrodes. The coatings were obtained by promoting the $\mathrm{Cu}(\mathrm{I})$-catalyzed click reaction between alkyne and azide moieties in the vicinity of the electrode by the electrochemical generation of $\mathrm{Cu}(\mathrm{I})$ ions. The physicochemical properties of the covalently reticulated hybrid films obtained were studied by SEM, AFM, UV-Vis and fluorescence spectroscopy. The one-pot covalent click reaction between the nanocapsules and the PEG linkers in the film did not affect the desirable features of the Hybridosomes ${ }^{\circledR}$ i.e. their hollow nanostructure their chemical versatility and their $\mathrm{pH}$-sensitivity. Consequently, both the composition and the cargo-loading of Hybridosomes ${ }^{\circledR}$ films could be tuned, demonstrating the versatility of these hybrid coatings. As an example, Hybridosome ${ }^{\circledR}$ films were used to encapsulate and release a bodipy fluorescent probe in response to either a $\mathrm{pH}$ drop or the application of an oxidative $+1 \mathrm{~V}$ potential $(v \mathrm{Ag} / \mathrm{AgCl}$ ) at the substrate. By bringing the field of electro-synthesized films a step further toward the design of complex physicochemical interfaces, these results open perspectives for multifunctional coatings where a chemical versatility, a controllable stability and a hollow nanostructure are required.
\end{abstract}

\section{Introduction}

Over the past several decades, the continuous development of smart and active interfaces has highlighted the requirement for multifunctional coatings. This has triggered the development of

† These authors contributed equally

Corresponding Authors: RYDZEK.Gaulthier@nims.go.jp; flavien.sciortino@univ$\underline{\text { rennes1.fr }}$

a University of Rennes, Centre National de la Recherche Scientifique (CNRS, France), Institut des Sciences Chimiques de Rennes (ISCR), UMR 6226, F-35000 Rennes, France

${ }^{b}$ World Premier International (WPI) Research Center for Materials Nanoarchitectonics (MANA), National Institute for Materials Science (NIMS), 1-1 Namiki, Tsukuba 3050044, Japan

${ }^{c}$ CNRS UMI 3629 CNRS - Saint Gobain - NIMS, Laboratory for Innovative Key Materials and Structures (LINK), National Institute for Materials Science (NIMS), 1-1 Namiki, Tsukuba 305-0044, Japan

${ }^{d}$ Laboratoire de Chimie de Coordination UPR8241 CNRS, 205 rte de Narbonne, 31000 Toulouse Cedex 04, France.

${ }^{e}$ Graduate School of Frontier Sciences, The University of Tokyo, Kashiwa 277-0827, Japan

Electronic Supplementary Information (ESI) available: TEM Tomography, NTA scattering, NMR, ATR-FT IR spectroscopy, UV-Visible spectroscopy, S(T)EM, EDX CV, AFM and fluorescence spectroscopy analysis. See DOI: 10.1039/x0xx00000x several deposition strategies including Layer-by-Layer films, ${ }^{1}$ selfassembled monolayers, ${ }^{2,3}$ and various electro-synthesis technics ${ }^{4-6}$ with applications in fields including biomaterials ${ }^{7}$ and energy storage. ${ }^{8}$ With this respect, two main challenges have emerged: achieving the one-pot deposition of coatings and controlling their structure at the nanoscale. ${ }^{9-12}$ For instance, films containing nanocapsules have attracted a significant interest as they allow developing biomaterials, ${ }^{13}$ sensors, ${ }^{14}$ and energy storage devices. For this latter application, the use of porous capsules deposited over electrodes enables to accomodate for the large volume change accompagnying the electrochemical cycles. ${ }^{15}$ In the case of delivery devices, the surface trapping of intact vesicles or nanocapsules remains a challenge to preserve their cargo. ${ }^{16}$ Indeed, membrane rupture may occur, and in this regard the mechanical properties of the capsule are important. For applications that rely on the amount of released substances, such as drug delivery or sensors, improving the loading capacity of the film is beneficial. To this aim, 3D constructs obtained by direct immobilization of capsules are desirable. ${ }^{17}$ Early attempts included the layer-by-layer deposition of cerasome and liposome-containing films. ${ }^{18-20}$ Here we report on the one-pot construction of hybrid hollow nanocapsule films, that are able to release their encapsulated cargo in response to either a $\mathrm{pH}$ or an electrochemical stimulus. The recently reported $100 \mathrm{~nm}$ diameter hybrid nanocapsules, named Hybridosomes ${ }^{\circledR},{ }^{21,22}$ were used as hollow building blocks for assembling the film by 
reticulation via a localized coupling reaction. Possessing an hybrid shell composed of $5 \mathrm{~nm}$ inorganic nanoparticles (NPs) crosslinked by polyacrylic acid (Figure 1a, S-1 and Video S-1), Hybridosomes represent a new class of hollow nanocarriers with several levels of versatility arising from the individual characteristics of their organic and inorganic components and from their hollow structure. For instance, these versatile nanocapsules can be prepared from iron oxide nanoparticles (IONPs), gold nanoparticles, Quantum Dots (QDs) and their mixtures. The polymer crosslinkers in hybridosomes can be chemically modified to append a chemical recognition or reactive function. These capsules exhibit a narrow size distribution (ca $100 \mathrm{~nm}$ in diameter) and their core can be used to encapsulate susbtances. In addition, they exhibit outstanding mechanical properties, combining deformability and memory shape recovery. ${ }^{23}$ To assemble the film in one pot, Hybridosomes bearing alkyne moieties (functional polyacrylic acid PAA-C $\equiv \mathrm{CH}$ ) were reticulated by electro-click reaction with azide bearing homobifunctional polyethyleneglycol $\left(\mathrm{N}_{3}-\mathrm{PEG}-\mathrm{N}_{3}\right.$ ) linkers on $\mathrm{F}-\mathrm{SnO}_{2}$ (FTO) electrodes. A poly(ethyleneimine)-alkyne ( $\mathrm{PEI}-\mathrm{C} \equiv \mathrm{CH}$ ) pre-coating layer was used to promote the film buildup. This film assembling process, illustrated on Figure $1 \mathrm{~b}$, was performed by electrochemically generating $\mathrm{Cu}(\mathrm{l})$ ions, allowing the occurrence of a $\mathrm{Cu}(\mathrm{I})$-catalyzed Alkyne-Azide Click reaction (CuAAC) between the building blocks. ${ }^{24-}$ ${ }^{26}$ Such an "electro-click" reaction allows the construction of polymeric, ${ }^{26}$ organic ${ }^{27}$ and inorganic ${ }^{28}$ films on electrodes, including with supramolecular interactions ${ }^{29}$ and with pattern control. ${ }^{30,31}$ In this context, the physicochemical properties of electro-clicked Hybridosomes coatings are bringing the field of electro-synthesized films a step further, by enabling performing molecular encapsulation and subsequent stimuli-responsive release.

\section{Results and discussion}

2.1 Proof of concept. Both Hybridosome ${ }^{\circledR}$ nanocapsules and electro-click chemistry are emergent concepts useful for providing functional yet versatile materials. ${ }^{21,26}$ Hybridosomes are a new class of hybrid nanocapsules that combine the features of both the inorganic nanoparticles and the polymer chains contained in their shells. Here Hybridosomes based on maghemite IONPs and PAA$\mathrm{C} \equiv \mathrm{CH}$ have been used as clickable building blocks. ${ }^{32}$ In our previous work, such Hybridosomes inherited magnetic properties from their IONP components, facilitating their purification. ${ }^{23}$ Electro-click chemistry is now a recognized method for achieving a spatially confined covalent reaction between both organic and inorganic functional building blocks, resulting in the formation of engineered interfaces. ${ }^{28,33-36}$ The concept introduced here is based on the assembly of Hybridosome nanocapsules into covalent coatings by using the electro-click approach, while preserving the remarkable features of Hybridosomes. A $6 \%$ alkyne functionalized polyacrylic acid (PAA-C $\equiv \mathrm{CH}$ ) (synthesis and characterization of functional polymers is described in Schemes S-1, S-2 and Figure S-2) was used to decorate Hybridosomes with click-suitable moieties. The resulting nanocapsules were covalently coupled with azide homobifunctionnal PEG linkers $\left(\mathrm{N}_{3}-\mathrm{PEG}-\mathrm{N}_{3}\right)$ in the vicinity of a PEI-
$\mathrm{C} \equiv \mathrm{CH}$ pre-coated FTO electrode (Figure $1 \mathrm{~b}$ ). The local catalysis of the alkyne-azide Huisgen cycloaddition reaction was made possible by locally generating $\mathrm{Cu}(\mathrm{I})$ ions from $\mathrm{Cu}(\mathrm{II})$ in solution, by using cyclic voltammetry (CV). This process resulted in the construction of films composed of Hybridosome nanocapsules covalently reticulated with PEG linkers.

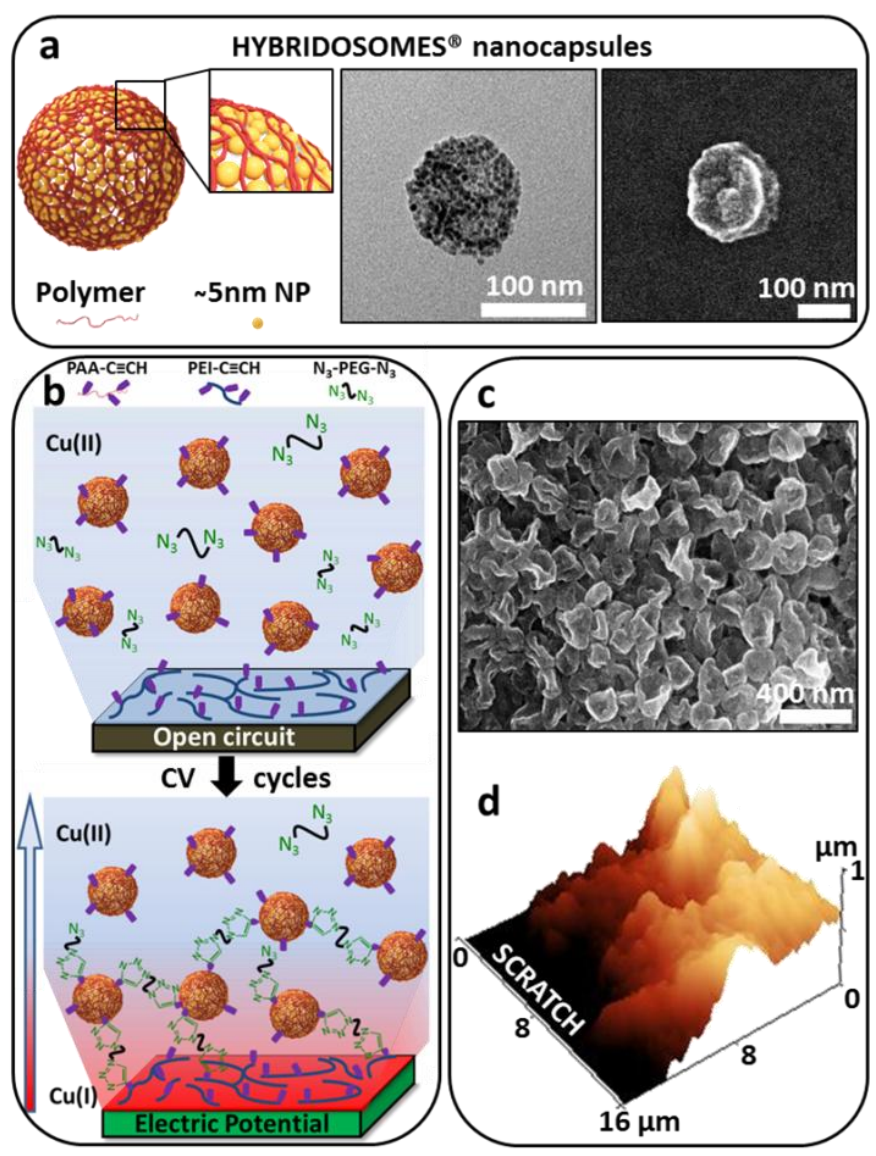

Figure 1: Concept of Hybridosomes ${ }^{\circledR}$ films covalently assembled by using electro-click chemistry. a) Schematic depiction, TEM and SEM of deflated Hybridosome nanocapsules composed of iron oxide nanoparticles (IONPs) and polyacrylic acid. b) Schematic representation of the electro-click process, allowing the construction of films on $\mathrm{PEI}-\mathrm{C} \equiv \mathrm{CH}$ pre-coated electrodes, by using alkyne-functionalized Hybridosomes and azide homobifunctionnal PEG linkers as building blocks in the presence of electrogenerated $\mathrm{Cu}(\mathrm{I})$ catalyst. c) SEM micrograph of a typical Hybridosomes film in the dried state. The film was constructed by electroclick after $800 \mathrm{CV}$ cycles $(-0.2 \mathrm{~V}$ to $0.6 \mathrm{~V} v \mathrm{sg} / \mathrm{AgCl}, 50 \mathrm{mV} / \mathrm{s})$ in the presence of $4.5 \times 10^{9}$ Hybridosomes $/ \mathrm{mL}, 0.1 \mathrm{mg} / \mathrm{mL} \mathrm{N}$-PEG-N $\mathrm{N}_{3}$ and $0.6 \mathrm{mM} \mathrm{CuSO}_{4}$ at $\mathrm{pH}$ 3.5. d) Typical 3D view of the corresponding scratched film measured by AFM in the dry state and contact mode. 


\section{PCCP}

\section{ARTICLE}
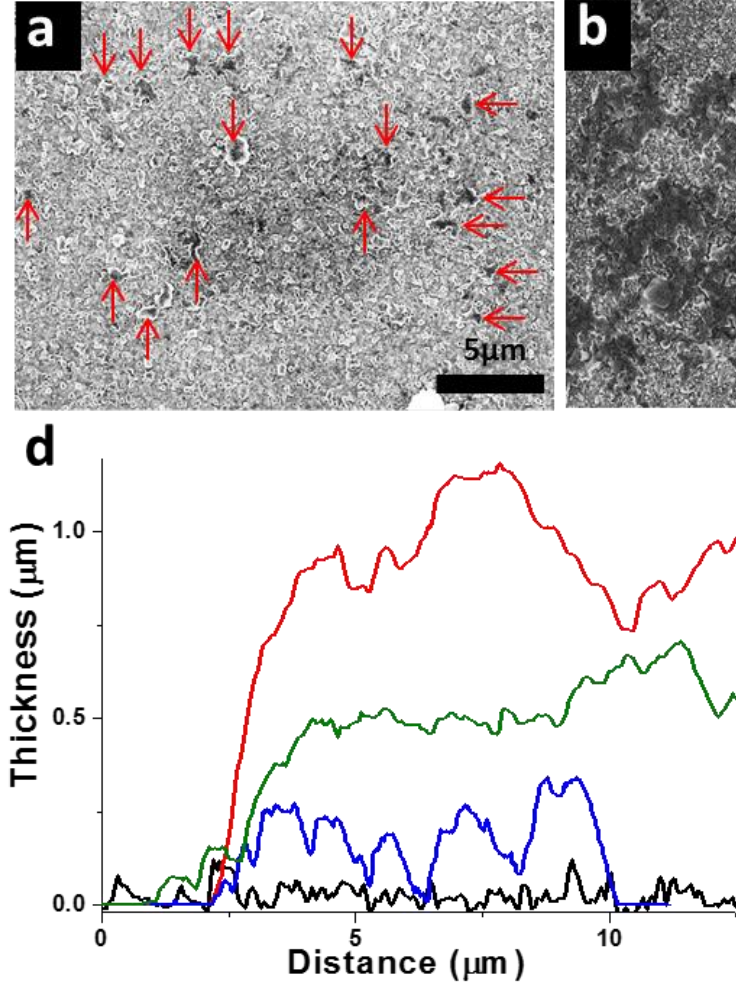
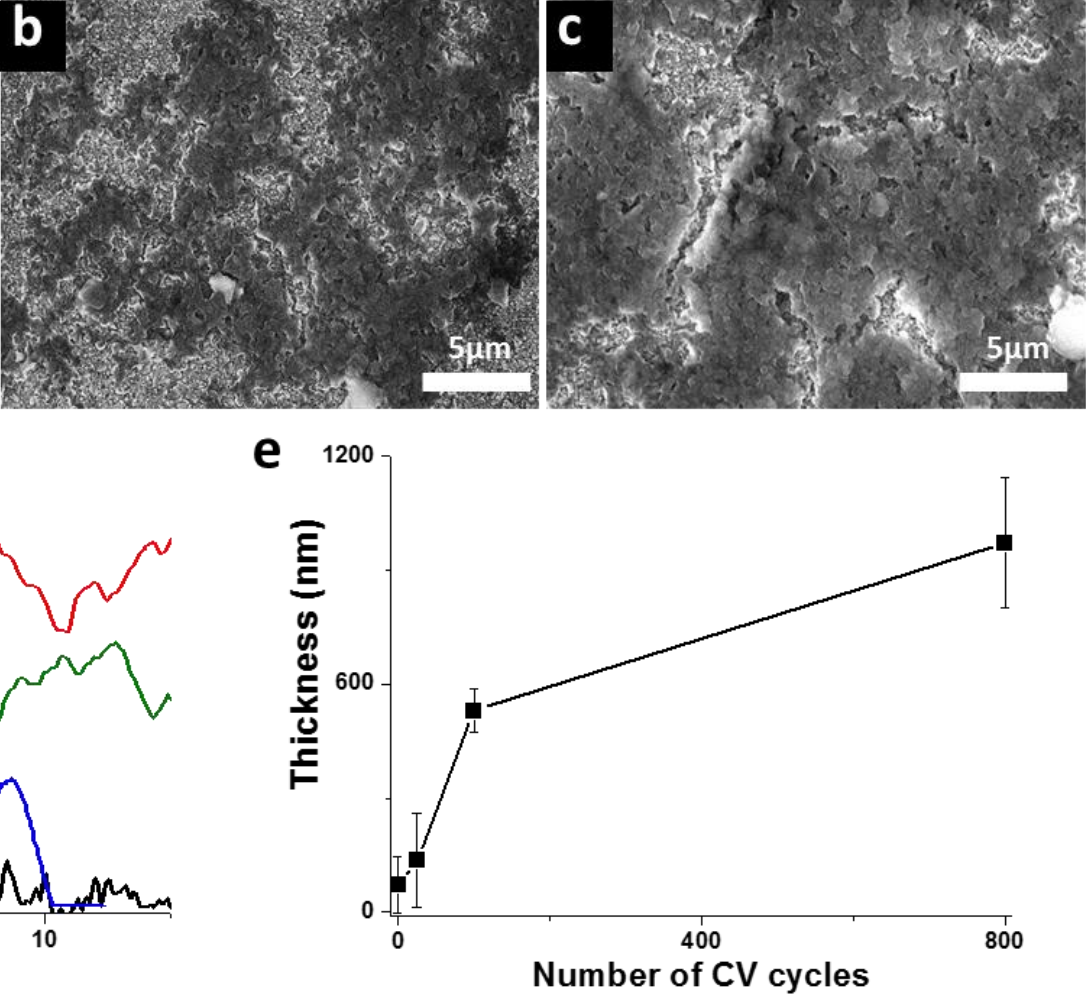

Figure 2: Germination and growth of covalently reticulated Hybridosomes ${ }^{\circledR}$ films. $\quad$ SEM micrographs of Hybridosome films on FTO after 25 (a), 100 (b) and 800 (c) CV cycles (-0.2 V to $0.6 \mathrm{~V}$ vs Ag/ $\mathrm{AgCl}, 50 \mathrm{mV} / \mathrm{s}$ ) in the presence of $4.5 \times 10^{9} \mathrm{Hybridosomes} \mathrm{mL}, 0.1 \mathrm{mg} / \mathrm{mL} \mathrm{N}-\mathrm{PEG}-\mathrm{N}_{3}$ and $0.6 \mathrm{mM} \mathrm{CuSO}{ }_{4}$ at pH 3.5 . d) Cross-sectional profiles of Hybridosome films, measured by AFM in the dry state and contact mode, after 0 (black line), 25 (blue line), 100 (green line) and 800 (red line) CV cycles. e) Evolution of the thickness of the corresponding films in the dry state

Scanning electronic microscopy (SEM) investigations on the resulting electrodes reveal the stacking of several nanocapsules, forming a film (Figure 1c) whose thickness, measured by atomic force microscopy (AFM) in the dried state, exceeded $850 \mathrm{~nm}$ after $800 \mathrm{CV}$ cycles (Figure 1d). Such a thickness value corresponds approximately to the stacking of 10 layers of Hybridosomes since dried nanocapsules, as seen by AFM, are approximately $70 \mathrm{~nm}$ thick. To demonstrate that this film construction can be attributed to the occurrence of the alkyne-azide Huisgen cycloaddition between the functional building blocks, control experiments were performed (Figure S-3). When one component required for the click reaction was removed, i.e. either the copper ions, the $\mathrm{N}_{3}-\mathrm{PEG}-\mathrm{N}_{3}$ linkers or the $\mathrm{CV}$ required to generate the $\mathrm{Cu}(\mathrm{I})$ catalyst, no film construction was observed. This implies that the coating growth originates from the covalent "click" reaction between functional Hybridosomes and $\mathrm{N}_{3}$-PEG- $\mathrm{N}_{3}$ linkers. ${ }^{26}$ To further confirm this trend, Attenuated Total Reflectance Fourier-Transform InfraRed spectroscopy (ATR-FTIR) was performed on the film and compared with the spectrum of its organic components, i.e. the $\mathrm{N}_{3}-\mathrm{PEG}-\mathrm{N}_{3}$ linkers, the PAA-C $\equiv \mathrm{CH}$ from clickable Hybridosomes and the PEI$\mathrm{C} \equiv \mathrm{CH}$ from the pre-coating layer. The spectrum of electro-clicked Hybridosome coatings exhibited typical absorption bands of all these components, confirming their inclusion in the film (Figure S4). Interestingly, the intense absorption peak of azide groups, visible on the spectrum of $\mathrm{N}_{3}-\mathrm{PEG}-\mathrm{N}_{3}$ at $2100 \mathrm{~cm}^{-1}$, was absent from the spectrum of the film, contrary to the absorption peaks of ethylene oxide group of PEG at $1060 \mathrm{~cm}^{-1}$, further confirming the occurrence of the click reaction. ${ }^{25,37}$

2.2 Hybridosome ${ }^{\circledR}$ films construction and growth mechanism. The growth mechanism of IONP-based Hybridosome films was investigated by SEM microscopy after 25, 100 and $800 \mathrm{CV}$ cycles (Figure 2). At each stage, a rough evaluation of the film surface coverage was performed by thresholding the micrographs. Several germination points emerged at early growth stages, achieving a surface coverage of around $11 \%$, as calculated from Figure 2 a. When the buildup was allowed proceeding further, the film growth 


\section{PCCP}

ARTICLE

achieved percolation, reaching a surface coverage of $75 \%$ after 100 CV cycles (calculated from Figure 2b) and above $90 \%$ after 800 cycles (calculated from Figure $2 \mathrm{c}$ ). In addition to this topological evolution, the thickness of the film increased to $850 \mathrm{~nm}$ after 800 $\mathrm{CV}$ cycles (Figure $2 \mathrm{~d}$, e and S-5). The roughness of the films was estimated by calculating the RMS from AFM data of the film thickness on covered areas (table S-1). High roughness values were obtained which seem to correlate with the use of bifunctional PEG linkers, as it was observed in our previous study and by El Haitami et al. $^{27,38}$ When Hybridosomes were adsorbed on the PEI-C $\equiv \mathrm{CH}$ coated electrode (0 CV cycles), the surface topography exhibited nodes around $70 \mathrm{~nm}$ thick corresponding to the thickness previously measured by AFM for single dried Hybridosomes (Figure $2 \mathrm{~d}$, black line). Single Hybridosomes seem thus able to adsorb on the PEI$\mathrm{C} \equiv \mathrm{CH}$-coated electrode in the absence of electro-click reaction without leading neither to any significant surface coverage nor to the stacking of several nanocapsules (Figure S-3c). In contrast, when electro-click construction was performed for $25 \mathrm{CV}$ cycles, the emergence of 200-300 $\mathrm{nm}$ thick structures was observed, suggesting that stacks of to three to four layers of Hybridosomes already formed (Figure 2d, blue line). After a larger number of electro-click CV cycles, both the surface coverage and the thickness of the coating increased, reaching respectively $90 \%$ and $850 \mathrm{~nm}$ after $800 \mathrm{CV}$ cycles in the dried state (Figure $2 \mathrm{~d}$, green and red lines). At the same time, the intensity of the oxidation peak of copper on the electro-click voltamogram decreased with the number of cycles, indicating the insulating nature of the film, as expected for a polymer/maghemite composite film (figure S-5). These results are in accordance with previous studies where the electro-click construction relied exclusively on polymers. This confirms that the range of building blocks that can be used for assembling electro-clicked films can be extended to Hybridosomes, leading to both the germination and growth of films based on multiple layers of covalently reticulated nanocapsules with both a tunable surface coverage and thickness.

2.3 Preservation of Hybridosomes $^{\circledR}$ properties in the film. Hybridosomes are promising building blocks for designing materials due to their chemical versatility ${ }^{32}$, their hybrid polymer/NP composition and their hollow structure. Preserving these features when Hybridosomes are integrated in electro-clicked coatings is thus desirable. The stability of the nanocapsules against the electrochemical conditions used for assembling the films was investigated by applying a CV to an IONPs-based Hybridosomes dispersion. The absence of any faradic current measured in the ($0.2 \mathrm{~V}$ to $0.6 \mathrm{~V}$ ) CV window indicated the electrochemical stability of Hybridosomes (Figure S-7a). The structural stability of these building blocks was investigated by measuring the size-distribution of nanocapsules by SEM and scanning transmission electronic microscopy (STEM), both before and after their incorporation in the films (Figure S-7b). The size distribution of Hybridosomes did not significantly change after their incorporation in electro-clicked coatings with an average diameter increasing from $114 \mathrm{~nm} \mathrm{(+/-} 22$ $\mathrm{nm})$ to $122 \mathrm{~nm}(+/-21 \mathrm{~nm})$. Hybridosomes building blocks thus exhibit good structural tolerance to the electro-click synthesis conditions, leading to hybrid films with a hollow nanostructure. The chemical composition of IONPs-based Hybridosome films was determined by using Energy Dispersive X-ray (EDX) spectroscopy (Figure S-8). Detection of oxygen, carbon, nitrogen and iron indicate the presence of the polymeric (PAA-C $\equiv \mathrm{CH}, \mathrm{PEI}-\mathrm{C} \equiv \mathrm{CH}, \mathrm{N}_{3}-\mathrm{PEG}-\mathrm{N}_{3}$ ) and inorganic (IONPs) components in the film. The hybrid nature of electro-clicked Hybridosomes films was thus confirmed. Since Hybridosomes can be synthesized by using a large range of NPs, ${ }^{21}$ the transferability of this chemical versatility to electro-clicked films was investigated. The possibility to assemble nanocapsule films based on a different choice of NPs would indeed open perspectives in several fields of applications including catalysis, ${ }^{40-42}$ energy storage ${ }^{10,43}$ optoelectronic, ${ }^{44}$ sensing ${ }^{45}$ and controlled release ${ }^{21,39}$ As an example, another type of Hybridosome has been synthesized by using CdSe@ZnS QD, IONPs and PAA-C $\equiv \mathrm{CH}$ as building blocks. The resulting Hybridosomes exhibited the same morphology as nanocapsules entirely composed of IONPs. However, as expected, their EDX analysis indicates the presence of $\mathrm{Zn}, \mathrm{Se}, \mathrm{Cd}$ and $\mathrm{S}$ elements contained in the QDs (Figure 3a). When such Hybridosomes were used as building-blocks in the electro-click process, the coatings obtained presented similar morphological properties as films assembled from IONPs-based Hybridosomes (Figure $3 b$ ). Chemical analysis of these films, by using EDX, revealed the presence of $\mathrm{Fe}, \mathrm{O}, \mathrm{Zn}, \mathrm{Se}, \mathrm{S}$ confirming the inclusion of both IONPs and CdSe@ZnS QDs in the coating. These results demonstrated how the films can inherit the chemical versatility of Hybridosomes and their nanostructural features. 

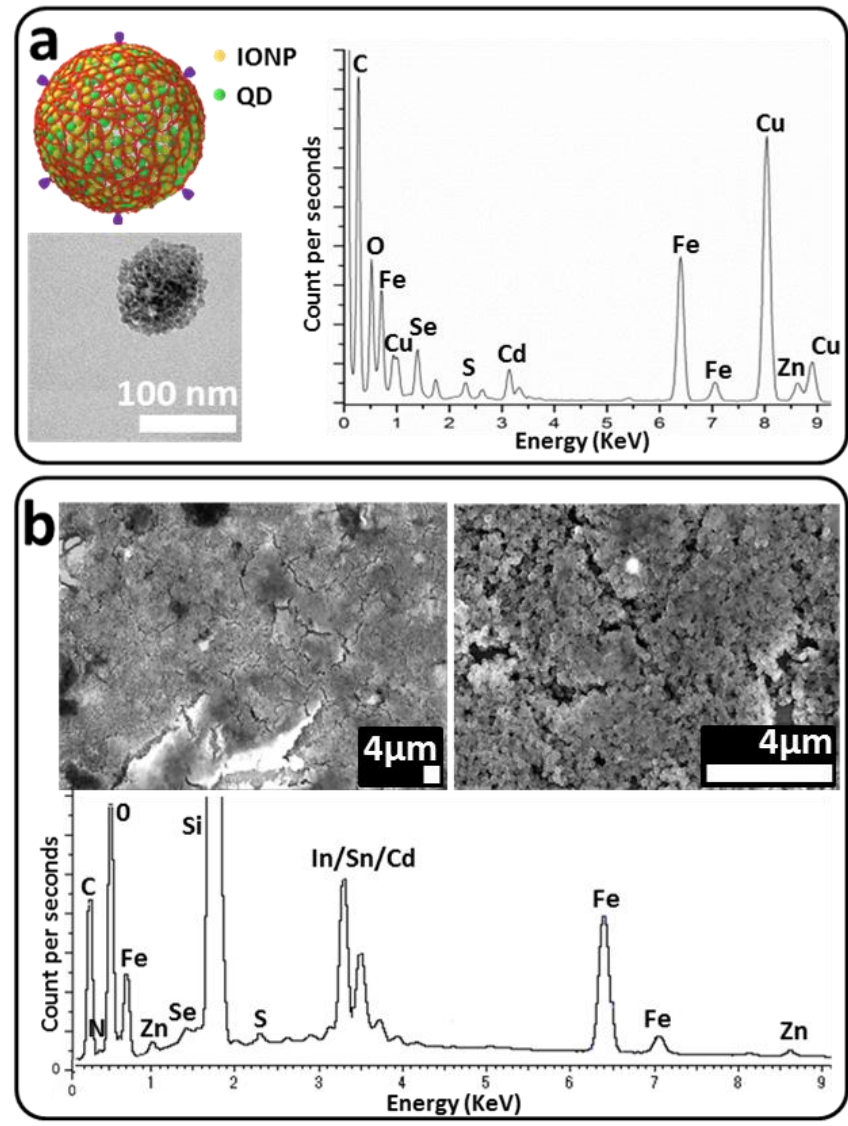

Figure 3: Chemical composition of films based on QD-containing Hybridosomes $^{\circledR}$. a) Schematic depiction, TEM micrograph and corresponding EDX analysis of Hybridosome nanocapsules composed of CdSe@ZnS quantum dots (green), IONPs (yellow) and PAA-C $\equiv \mathrm{CH}$ (red). b) SEM micrographs and EDX analysis of the corresponding films obtained after $800 \mathrm{CV}$ cycles $(-0.2 \mathrm{~V}$ to $0.6 \mathrm{~V}$ vs $\mathrm{Ag} / \mathrm{AgCl}, 50 \mathrm{mV} / \mathrm{s})$ in the presence of $4.5 \times 10^{9}$ Hybridosomes/mL, $0.1 \mathrm{mg} / \mathrm{mL} \mathrm{N}_{3}-\mathrm{PEG}-\mathrm{N}_{3}$ and $0.6 \mathrm{mM} \mathrm{CuSO}_{4}$ at $\mathrm{pH} 3.5$.

2.4 Encapsulation abilities of Hybridosomes ${ }^{\circledR}$. The preservation of the Hybridosomes nanostructure at the interior of electro-clicked films suggests applications in several fields including encapsulation and release of hydrophobic compounds. ${ }^{21}$ As a proof of concept, a fluorescent bodipy probe was encapsulated into Hybridosomes during their synthesis. The UV-visible absorbance spectrum of the resulting bodipy-loaded Hybridosomes dispersion was compared with the spectrum of both bodipy and empty Hybridosomes dispersions in water (Figure S-9a). Both bodipy-containing samples exhibited a similar UV-Vis absorption spectrum marked by the emergence of two prominent absorbance bands localized at $502 \mathrm{~nm}$ and $532 \mathrm{~nm}$. These peaks were absent from the spectrum of empty Hybridosomes dispersions, suggesting that the encapsulation process was successful and did not significantly modify the chemical properties of the probe. The fluorescence properties of bodipyloaded Hybridosomes dispersions were compared with the spectra of bodipy in a good solvent (Tetrahydrofuran, THF) and in water where bodipy is poorly soluble (Figure S-9b). A single fluorescence peak localized at $540 \mathrm{~nm}$ was observed for well-dissolved bodipy in THF. On the contrary, the spectrum of bodipy in water was marked by the emergence of 2 broad emission peaks at $560 \mathrm{~nm}$ and 625 $\mathrm{nm}$, indicating the formation of J-aggregates. ${ }^{46}$ Interestingly the spectrum of bodipy-loaded Hybridosomes dispersions exhibited a main peak around $540 \mathrm{~nm}$ and a shoulder around $565 \mathrm{~nm}$, demonstrating that the encapsulation process largely inhibits aggregation of bodipy (Figure S-9b). The loading efficiency of bodipy in hybridosomes was estimated at ca 73 \% from absorbance measurements at $540 \mathrm{~nm}$ (figure $\mathrm{S}-10$ ). From this measurement, we calculate roughly that the stoichiometry is of 3 encapsulated Bodipy molecules for $1 \mathrm{Fe}$ atom. Importantly, when bodipy-loaded hybridosomes underwent further washings, the supernatant was completely clear of bodipy, indicating that the encapsulated dye does not leak spontaneously.

2.5 Features of bodipy-encapsulated Hybridosome ${ }^{\circledR}$ films. Using bodipy-loaded Hybridosomes instead of "empty" nanocapsules for assembling the films did not change the morphology of the obtained coatings (Figure 4a). To probe further the integrity of the Hybridosomes integrated in electro-clicked films, the spectral features of bodipy-loaded Hybridosomes coatings were investigated in the dry state and compared with bodipy and bodipy-loaded nanocapsules drop-casted on FTO (Figure $4 b$ and $4 c$ ). On the one hand, the UV-Vis absorbance spectra of all films exhibited similar characteristics with two prominent peaks at $502 \mathrm{~nm}$ and $532 \mathrm{~nm}$, confirming the inclusion of bodipy in the films. Probe aggregation during drying was signaled by the appearance of a new absorption band at $680 \mathrm{~nm}$ (Figure 4b). On the other hand, the fluorescence spectra of these films exhibited significant differences (Figure 4c). The spectrum of drop-casted bodipy films was comparable to the emission spectrum of bodipy in water, confirming that aggregation already was occurring in solution. However, bodipy-loaded Hybridosomes films, either drop-casted or electro-clicked, exhibited a broad emission band around $565 \mathrm{~nm}$, corresponding to the aggregate component already observed on the spectrum of bodipyloaded Hybridosomes dispersions (Figures $4 c$ and S-9b). Free bodipy was thus absent from Hybridosomes films, suggesting the absence of bodipy leaking from the nanocapsules during the electro-click process. These results confirm that the electro-click process with bodipy-loaded Hybridosomes not only preserves the nanocapsules integrity but also its cargo content. The relationship between the composition of the building mixture and the resulting electro-clicked films was investigated by mixing both empty $(E)$ and bodipy-loaded $(L)$ Hybridosome batches at different ratios. When the L/E ratio increased from $0 \%$ (pure empty Hybridosomes) to 10 $\%, 50 \%$ and $90 \%$, a fluorescence emission peak emerged and increased in intensity between 660 and $670 \mathrm{~nm}$, demonstrating the presence of increasing quantities of bodipy in the films (Figure $4 \mathrm{~d}$ ). 


\section{PCCP}

\section{ARTICLE}
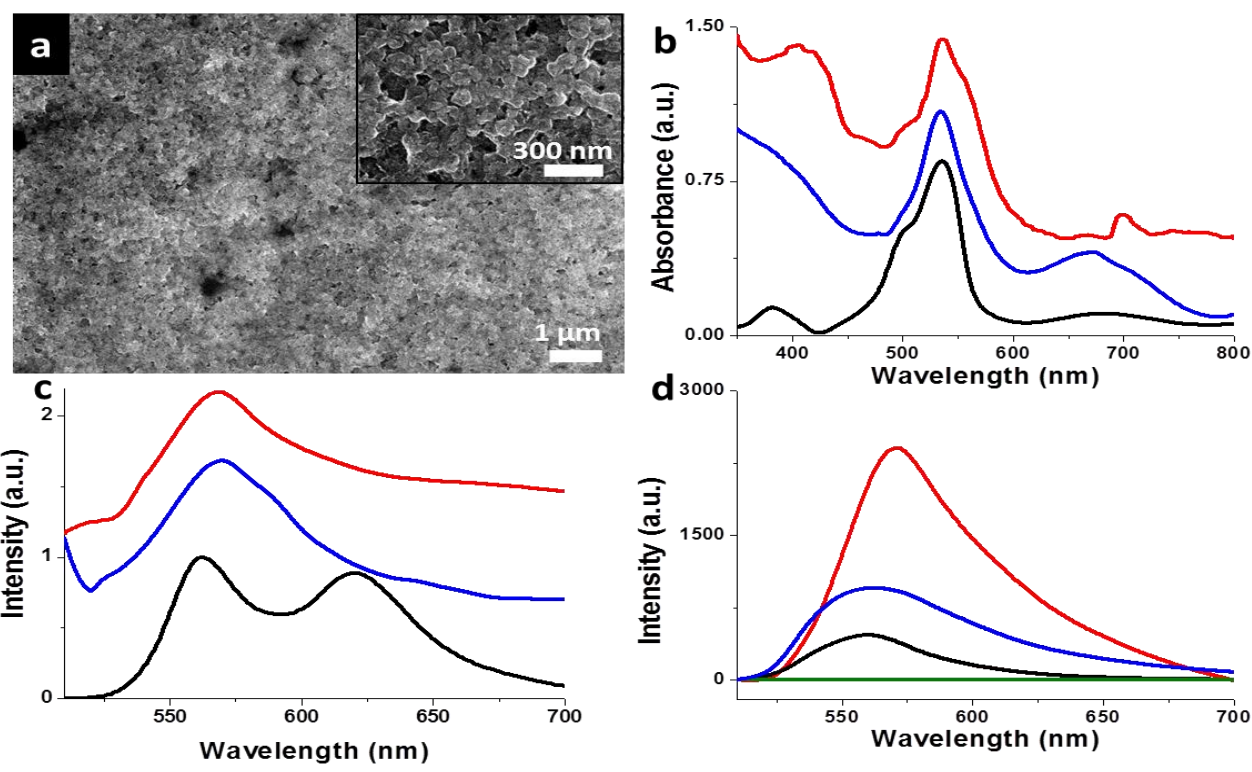

Figure 4: Bodipy-encapsulating Hybridosome ${ }^{\circledR}$ films. a) Typical SEM micrographs of bodipy-loaded Hybridosome films after 800 CV cycles. (b) UV-Visible absorbance spectra and (c) fluorescence spectra in the dry state $\left(\lambda_{\text {exc }}\right.$ at $480 \mathrm{~nm}$ ) of bodipy (black line), drop-casted bodipy-loaded Hybridosome (blue line) and an electro-clicked film based on bodipy-loaded Hybridosomes (red line). d) Fluorescence spectra in the dry state $\left(\lambda_{\text {exc }}\right.$ at $480 \mathrm{~nm}$ ) of electro-clicked Hybridosome films obtained from building mixtures containing $0 \%$ (green line), 10\% (black line), $50 \%$ (blue line) and $90 \%$ (red line) of bodipy-loaded Hybridosomes.

The area of the corresponding peaks increased accordingly, suggesting the absence of quenching when the L/E ratio was increased (Figure S-11). The interaction of the fluorophore with itself seems thus to remain constant, supporting the hypothesis that the probe is encapsulated inside separated compartments within the film. ${ }^{47}$ The simultaneous incorporation of several types of nanocapsules in the films, with a tunable ratio, seems thus possible. Hybridosome electro-clicked films constitute therefore a promising candidate for designing multiply-loaded coatings and multifunctional interfaces.

2.6 Stimulus-induced destabilization of Hybridosomes ${ }^{\circledR}$ films. The stability of electro-clicked nanocapsules was probed by using $\mathrm{pH}$ drops as an external stimulus. The effect of decreasing the $\mathrm{pH}$ from $\mathrm{pH} 4$ to $\mathrm{pH} 1$ was first studied on Hybridosomes dispersions and resulted in a two-step destabilization. At pH 3, many nanocapsules were destabilized and were reorganized into larger structures up to several microns in size (Figure S-12). $\mathrm{pH} 3$ coincides with the full protonation point of carboxylic groups of PAA chains, causing decreased polymer solubility in water, and reducing its ability to act as a colloidal stabilizer. This effect was recently reported as being responsible for the aggregation of PAA-coated silver and $\mathrm{TiO}_{2}$ nanoparticles at acidic $\mathrm{pH} .{ }^{48,49}$ This destabilization of Hybridosomes dispersions at $\mathrm{pH} 3$ illustrates how these nanocapsules inherit the properties of their polymer component. ${ }^{21}$ When the $\mathrm{pH}$ of Hybridosomes dispersions was decreased further to $\mathrm{pH} 1$, nanocapsules could no longer be observed and the morphology of the dispersed material dramatically changed (Figure S-12). Although IONPs composing the Hybridosomes are thermodynamically unstable at both $\mathrm{pH} 1$ and $\mathrm{pH} 3$, their dissolution kinetic is faster at $\mathrm{pH}$ 1. This illustrates how Hybridosomes also inherit properties of their inorganic components. ${ }^{50,51}$ The $\mathrm{pH}$-sensitivity of electro-clicked Hybridosome films was also investigated at $\mathrm{pH} \mathrm{3,2.5}$ and 1, confirming the trend observed in dispersion (Figure $\mathrm{S}-13$ ). Interestingly, the coatings were destabilized after soaking $15 \mathrm{~min}$ in a pH 2.5 solution instead of $\mathrm{pH} 3$ for nanocapsule dispersions. This suggests that the nanocapsules are better stabilized in the film environment. However, aggregated Hybridosomes were still clearly visible in the film at this $\mathrm{pH}$ value. In contrast, at $\mathrm{pH} 1$, the morphology of Hybridosome films observed by SEM was marked by the absence of nanocapsules, in agreement with results obtained with Hybridosomes dispersions. 


\section{PCCP}

\section{ARTICLE}

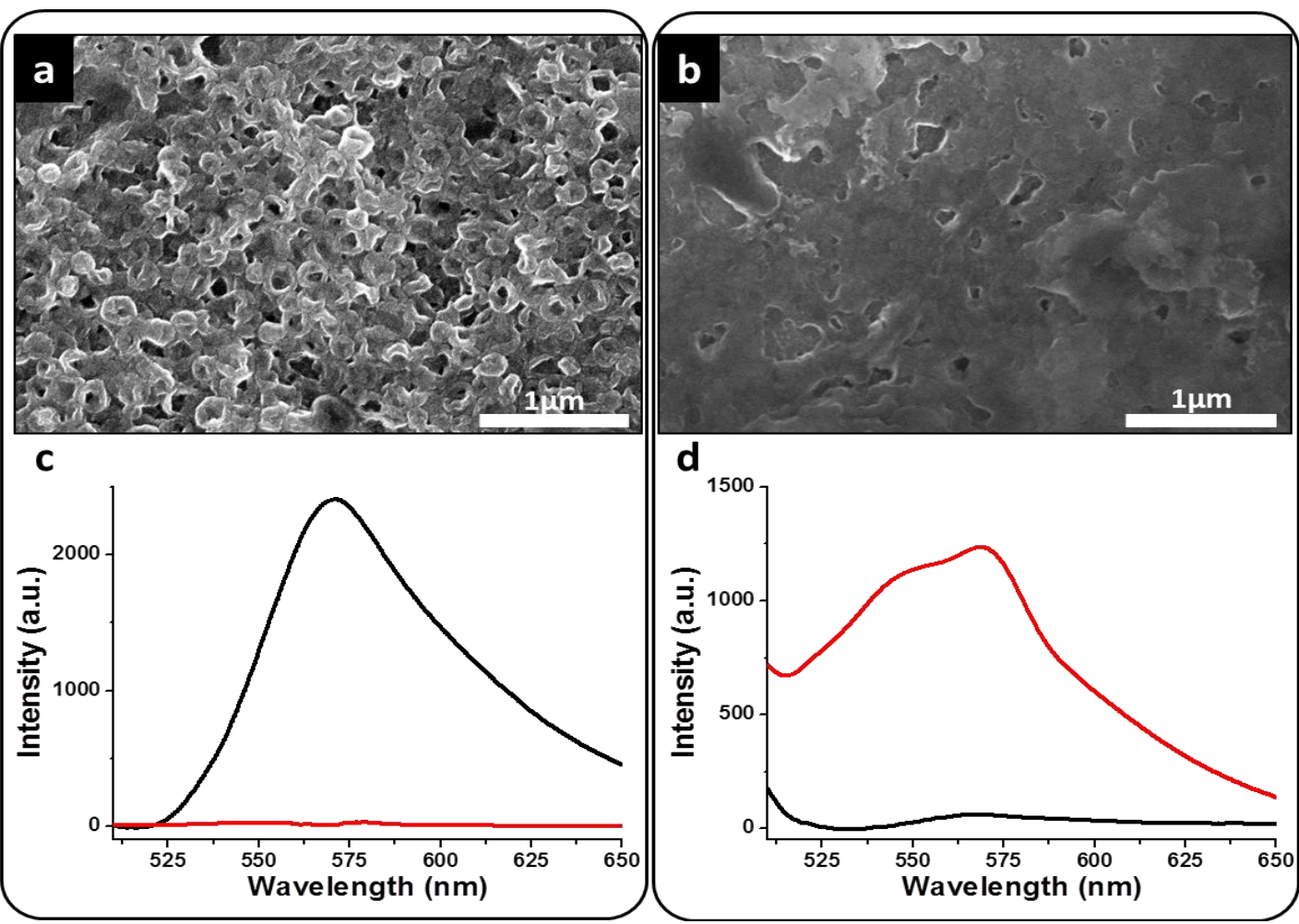

Figure 5: Electro-triggered release abilities of electro-clicked Hybridosomes ${ }^{\circledR}$ films. SEM micrographs $(a, b)$ and fluorescence spectra $\left(\lambda_{\text {exc }}\right.$ at $\left.480 \mathrm{~nm}\right)$ in the dry state (c, d) of bodipy-loaded Hybridosome films assembled on FTO (black line) and of the corresponding supernatant (red line) before (a,c) and after (b,d) application of $+1 \mathrm{~V}$ potential $(v \mathrm{sg} / \mathrm{AgCl})$ for 15 minutes in a $0.1 \mathrm{M} \mathrm{NaCl}$ solution.

2.7 Destabilization of bodipy-loaded Hybridosome ${ }^{\circledR}$ films. When bodipy-loaded nanocapsule films were brought into contact with a $\mathrm{pH} 1 \mathrm{HCl}$ solution, the partial dissolution of the coating was also observed (Figure S-14a and b). As a result, bodipy encapsulated in the film was released into the supernatant, which exhibited a fluorescence emission peak around $560 \mathrm{~nm}$ while the fluorescence of the film dramatically decreased (Figure S-14 c and d). By comparing the emission peak areas of the films before and after acidic treatment, the release rate of bodipy was estimated to exceed $95 \%$. This result demonstrates the ability of Hybridosome films to encapsulate and release molecules upon a direct $\mathrm{pH}$ change of the environment. Since these films are electro-clicked on conducting substrates, the possibility of destabilizing the coating by an electrochemically-induced reduction of local $\mathrm{pH}$ was also investigated (Figure 5). This approach, which consists of generating a proton gradient near the electrode by water electrolysis, has been reportedly used for assembly and dissolution of $\mathrm{pH}$-sensitive polymer films. ${ }^{52-54}$ Such a localized and easily controllable dissolution process is expected to trigger applications in the field of localized release of medical drugs, for instance by using implantable microelectrodes. ${ }^{55,56}$ A potential of $+1 \mathrm{~V}$ (vs $\left.\mathrm{Ag} / \mathrm{AgCl}, 0.1 \mathrm{M} \mathrm{NaCl}\right)$ was applied for $15 \mathrm{~min}$ to an electro-clicked Hybridosome film constructed on FTO in order to generate a proton gradient at the electrode. ${ }^{52}$ This treatment resulted in the destabilization of the coating as testified by the disappearance of nanocapsules from the surface at low SEM magnification (Figure $5 a$ and b). When higher magnifications were used, severely disorganized and fused Hybridosomes were visible on SEM micrographs, leading to the loss of their nanostructure (Figure S-15). This result was similar to the one obtained with Hybridosomes dispersions at $\mathrm{pH} 3$ and films soaked at pH 2.5 (Figure S-12 and S-13). Both the coated electrodes and their supernatant were studied by fluorescence spectroscopy $\left(\lambda_{\text {exc }}\right.$ at $\left.480 \mathrm{~nm}\right)$ prior to and following the electrochemical stimulus. Before water electrolysis, no fluorescence was measured in the supernatant while the coated electrode exhibited an emission peak centered at $568 \mathrm{~nm}$, indicating the presence of encapsulated bodipy (Figure 5c, black line). After $15 \mathrm{~min}$ of water electrolysis, the fluorescence intensity of the electrode dramatically decreased while the supernatant exhibited two broad emission peaks centered at 550 and $570 \mathrm{~nm}$ indicating the release of aggregated bodipy in the aqueous supernatant (Figure $5 \mathrm{~d}$ ). The release rate, estimated 


\section{PCCP}

ARTICLE

from the emission peak area of the film, exceeded 99\%. These two method for destabilizing electro-click Hybridosome films suggest their application in the directed release of encapsulated molecules in response to either a global $\mathrm{pH}$ change or localized water electrolysis.

\section{Conclusion}

Nanocapsule films composed of hollow PAA/IONPs nanocapsules and PEG linkers were assembled on FTO electrodes by electro-click cross-linking of organic/inorganic Hybridosomes ${ }^{\circledR}$. The growth of the film followed a germination/percolation mechanism, allowing tuning both the surface coverage (calculated from $520 \mu^{2}$ area micrographs) and the thickness of the films. After $800 \mathrm{CV}$ cycles, a surface coverage exceeding $90 \%$ and a film thickness over $850 \mathrm{~nm}$ were achieved. This work clearly demonstrates that the mild physicochemical conditions required by the electro-click approach do not alter the chemical and structural properties of Hybridosome nanocapsules. Consequently, both the composition and the loading of the nanocapsules could be changed, demonstrating the versatility and encapsulation abilities of Hybridosome films. As an example, a bodipy probe was successfully encapsulated in Hybridosomes and preserved upon the film construction. When a mixture of both bodipy-loaded and empty nanocapsules were used to assemble the film, the coating composition reflected the stoichiometry found in solution. The corresponding coatings could be destabilized by using either a $\mathrm{pH}$ or an electrochemical stimulus; releasing more than $95 \%$ of their fluorescent content within $15 \mathrm{~min}$. By bringing the field of electro-synthesized films a step further toward the design of complex physicochemical interfaces, these results open perspectives for multifunctional coatings where a chemical versatility, a controllable stability and a hollow nanostructure are required.

\section{Experimental}

Materials. Copper sulfate pentahydrate $\left(\mathrm{CuSO}_{4} \times 5 \mathrm{H}_{2} \mathrm{O}, \mathrm{M}=249.7\right.$ $\mathrm{g} / \mathrm{mol}$, CAS 7758-99-8), branched polyethyleneimine $(M=25 \mathrm{kDa}$, CAS 9002-98-6), Polyacrylic acid ( $M=450 \mathrm{kDa}$, CAS 9003-01-4), amino-EG ${ }_{4}$-alkyne $(M=231.3 \mathrm{~g} / \mathrm{mol}$, CAS 1013921-36-2), 10 undecynoic acid ( $M=182.3 \mathrm{~g} / \mathrm{mol}$, CAS 2777-65-3), polyoxyethylene bis(azide) $(M=2050 \mathrm{~g} / \mathrm{mol}$, CAS 82055-94-5), Benzotriazol-1yloxy)tris(dimethylamino)phosphonium hexafluorophosphate (BOP, $M=442.28 \mathrm{~g} / \mathrm{mol}$, CAS 56602-33-6), N,N-Diisopropylethylamine (DIEA, $M=129.24 \mathrm{~g} / \mathrm{mol}$, CAS 7087-68-5), N,N-Dimethylformamide (DMF, $M=73.09 \mathrm{~g} / \mathrm{mol}$, CAS 68-12-2), octadecylamine-coated CdSe@ZnS (product number 790192) were purchased from Sigma- aldrich and used as received. Tetrahydrofuran (THF, $M=$ $72.10 \mathrm{~g} / \mathrm{mol}$, CAS 109-99-9) was purchased from VWR. Hydrochloric acid $(\mathrm{HCl}, M=36.46 \mathrm{~g} / \mathrm{mol}$, CAS 7647-01-0), Sodium Hydroxide ( $\mathrm{NaOH}, M=39.99 \mathrm{~g} / \mathrm{mol}$, CAS $122000-64-5)$ were purchased from Wako. 4,4-Difluoro-8-(4'-trimethylsilylethynylphenyl)-1,3,5,7tetramethyl-2,6-diethyl-4-bora-3a,4a-diaza-s-indacene (Bodipy, $M=$ $476.2 \mathrm{~g} / \mathrm{mol}$ ) was kindly provided by $O$. Mongin (ISCR) and synthesized following a reported procedure. ${ }^{57}$ FTO electrodes were purchased from ALS, Japan. All aqueous solutions were prepared with MilliQ water $\left(18.2 \mathrm{M} \Omega . \mathrm{cm}^{-1}\right)$ purified using a Purelab Prima system.

Synthesis of functional polymers. Synthesis of PAA-C $\equiv C H$ was adapted from our previous work and entails grafting amino- $\mathrm{EG}_{4^{-}}$ alkyne on the polyacrylic acid backbone. ${ }^{58}$ PAA $(2 \mathrm{mmol})$ was dissolved in DMF $(7 \mathrm{~mL})$ with BOP $(142 \mu \mathrm{mol})$ under stirring for 10 $\mathrm{m}$. Amino-EG ${ }_{4}$-alkyne $(108 \mu \mathrm{mol})$ was dissolved in DMF $(5 \mathrm{~mL})$ and DIEA ( $2 \mathrm{mmol}$ ) was added. After 90 minutes, DMF was evaporated under reduced pressure. The residue was dissolved in milliQ water, dialyzed (Spectra/Por, MWCO 12kDa) against milliQ water for $48 \mathrm{~h}$ and recovered by evaporation under reduced pressure and freeze drying. Synthesis of PAA-C $\equiv \mathrm{CH}$ was performed by using an EDC/NHS approach. 10-Undecynoic acid $(0.2 \mathrm{mmol})$ was dissolved in dichloromethane $(7 \mathrm{~mL})$ with 3-fold molar excess of (1-ethyl-3-(3dimethylaminopropyl)carbodiimide hydrochloride and Hydroxysuccinimide. After 10 min stirring, PEI $(2 \mathrm{mmol})$ dissolved in dichloromethane $(7 \mathrm{~mL})$ were added and the reaction was allowed to proceed for 90 minutes. Dichloromethane was evaporated under reduced pressure. The residue was dissolved in milliQ water, dialyzed (Spectra/Por, MWCO $12 \mathrm{KDa}$ ) against milliQ water for $48 \mathrm{~h}$ and recovered by evaporation under reduced pressure and freeze drying. The functionalization degree of the obtained polymers was evaluated by using NMR spectroscopy (Supporting Information).

Preparation of Iron Oxide based Hybridosomes ${ }^{\circledR}$ and encapsulation step. Hybridosomes were elaborated as previously reported ${ }^{21}$ by using PAA-C $\equiv \mathrm{CH}$ and superparamagnetic iron oxide nanoparticles previously synthesized. ${ }^{59}$ In a typical process, THF $(100 \mu \mathrm{L})$ was added to a dispersion of iron oxide nanoparticles $\left(\mathrm{m}_{\mathrm{Fe}}\right.$ $=52.6 \mu \mathrm{g})$ followed by water $(800 \mu \mathrm{L})$ then stirred. After $24 \mathrm{~h}$, PAA$\mathrm{C} \equiv \mathrm{CH} \quad(2,1 \mathrm{mM})$ was added to the mixture before solvent evaporation for $15 \mathrm{~h}$ at $40^{\circ} \mathrm{C}$. The resulting precipitate was magnetically attracted and dispersed in milliQ water $(930 \mu \mathrm{L})$. The same procedure was used to synthesize mixed iron oxide (IONPs)/ quantum dots (QDs) Hybridosomes by initially mixing $50 \mu \mathrm{L}\left(\mathrm{m}_{\mathrm{Fe}}=\right.$ $26.3 \mu \mathrm{g})$ of iron oxide (IONPs) dispersion with $50 \mu \mathrm{L}\left(\mathrm{m}_{\mathrm{QD}}=25 \mu \mathrm{g}\right)$ of quantum dots (QDs) dispersion in $100 \mu \mathrm{L}$ of THF. Encapsulation of bodipy in Hybridosomes was performed by adding $100 \mu \mathrm{L}$ of a $1 \mathrm{mM}$ bodipy in THF into $50 \mu \mathrm{L}\left(\mathrm{m}_{\mathrm{Fe}}=26,3 \mu \mathrm{g}\right.$ ) of the initial iron oxide (IONPs) suspension. 


\section{PCCP}

Film construction. ITO and FTO electrodes were cleaned by dipping in $0.1 \mathrm{M} \mathrm{NaOH}$ and $0.1 \mathrm{M} \mathrm{HCl}$ baths for 15 minutes followed by rinsing. A PEI-C $\equiv \mathrm{CH}$ pre-coating layer was deposited by dipping (10 $\mathrm{mg} / \mathrm{mL}$ ) for 15 minutes and subsequent rinsing. The film was constructed byapplying a cyclic voltammetric current $(-200 \mathrm{mV}$ to $+600 \mathrm{mV}$ vs $\mathrm{Ag} / \mathrm{AgCl}$ at $50 \mathrm{mV}^{-1} \mathrm{~s}^{-1}$ under stirring) to the electrode in contact with a pH 3.5 solution containing typically $4.5 \times 10$ Hybridosomes $/ \mathrm{mL}, 0.6 \mathrm{mM} \mathrm{CuSO}_{4}$ and $0.1 \mathrm{mg} / \mathrm{mL} \mathrm{N}_{3}$-PEG- $\mathrm{N}_{3}$.

Film destabilization. Bodipy-loaded Hybridosomes films were first constructed by using $800 \mathrm{CV}$ cycles. The resulting coated electrodes were either dipped in an $\mathrm{HCl}$ solution at desired $\mathrm{pH}$ or subjected to a $+1 \mathrm{~V}$ potential ( $v s \mathrm{Ag} / \mathrm{AgCl}$, in a $0.1 \mathrm{M} \mathrm{NaCl}$ buffer) for 15 minutes. The supernatant was analyzed directly after the process. The electrodes were rinsed with Milli- $Q$ water and dried before analysis.

Cyclic Voltametry: A CHI model 613B potentiostat was used with a three-electrode apparatus based on an ITO and FTO coated quartz as working electrode, a platinum wire as counter electrode, and an RE1S $\mathrm{Ag} / \mathrm{AgCl}$-based reference electrode. The electrodes were purchased from ALS.

Fluorescence spectroscopy was performed using a JASCO FP8500 spectrofluorometer.

NMR analysis of functionalized polymers $\mathrm{PEI}-\mathrm{C} \equiv \mathrm{CH}$ and $\mathrm{PAA}-\mathrm{C} \equiv \mathrm{CH}$ were performed in $\mathrm{D}_{2} \mathrm{O}$ on a Bruker Avance III HD $500 \mathrm{MHz}$ spectrometer fitted with a Dual ${ }^{1} \mathrm{H} /{ }^{13} \mathrm{C}$ probehead.

Atomic force microscopy (AFM) was performed by using an AFM SPA400-SPI4000 (Seiko Instruments Inc., Chiba, Japan) in contact mode and in the dried state with silicon nitride cantilevers, spring constant $0.08 \mathrm{~N} / \mathrm{m}$ (model SN-AF01S-NTK-W10200326 from Seiko Instruments). Height images were scanned at a fixed scan rate of 1 $\mathrm{Hz}$. The thickness of PEM films was measured by imaging the coatings after scratching. When possible, the AFM scanning direction was perpendicular to the scratch. Data evaluation was performed by using the Gwyddion software. A plane-fit treatment was applied to the scratched area of each image, and its minimum height was set to $z=0$.

Scanning electron microscopy (SEM), scanning transmission electron microscopy (STEM) and energy-dispersive $\mathrm{X}$-ray spectroscopy (EDX) were performed using a Hitachi S-4800 at accelerating voltages of $30 \mathrm{kV}$. The samples were observed directly after 15 min drying under vacuum. Calculation of Hybridosomes size distribution analysis and film surface coverage was performed from SEM and STEM data by using the ImageJ software.

Transmission electron microscopy (TEM), energy-dispersive X-ray spectroscopy (EDS) and tomography were performed using a JEM-
2100 (JEOL) transmission electron microscope (accelerating voltage $200 \mathrm{kV}$ ) equipped with a CCD camera.

UV-visible spectroscopy was performed using a Shimadzu (Japan) UV visible NIR spectrophotometer (model UV-3600).

Attenuated total reflection infrared spectroscopy (ATR-FTIR) on Hybridosomes films and functionalized polymers was performed by using a Thermo Scientific Nicolet 4700 apparatus (USA).

Nanoparticle Tracker Analysis (NTA) tracks individual trajectories, allowing the calculation of the diffusion coefficient and thus of the hydrodynamic diameter of each particle. NTA was carried out with a Nanosight LM10 device system equipped with a $40 \mathrm{~mW}$ laser working at $\lambda=638 \mathrm{~nm}$. Video sequences were recorded via a CCD camera operating at 30 frames per second and evaluated via the NANOSIGHT NTA 2.0 Analytical Software Suite. The hybridosomes suspensions at $[\mathrm{Fe}] \sim 50 \mu \mathrm{g} / \mathrm{mL}$ are washed two times after magnetic separation and diluted 100 times before NTA analysis.

\section{Conflicts of interest}

There are no conflicts to declare.

\section{Acknowledgements}

This work was supported by the JSPS KAKENHI Grant Number JP16H06518 (Coordination Asymmetry) and CREST, JST. F. S. warmly thanks the Embassy of France at Tokyo and MAEDI for travel financial support and University Bretagne Loire and the Brittany region for daily allowance financial support. G.R. thanks Dr. Loic Jierry for fruitful discussions. The authors wish to acknowledge the financial support of the Centre National de la Recherche Scientifique (CNRS, France) and of the Ministère de l'enseignement Supérieur la Recherche et de l'Innovation (France). 


\section{PCCP}

\section{ARTICLE}

\section{References}

1 G. Decher, Science, 1997, 277, 1232-1237.

2 C. D. Bain and G. M. Whitesides, Science, 1988, 240, 62-63.

3 I. Rubinstein, S. Steinberg, Y. Tor, A. Shanzer and J. Sagiv, Nature, 1988, 332, 426-429.

4 C. Li, H. Bai and G. Shi, Chem. Soc. Rev., 2009, 38, 2397-2409.

5 S. E. Fosdick, K. N. Knust, K. Scida and R. M. Crooks, Angew. Chem. Int. Ed., 2013, 52, 10438-10456.

6 G. Rydzek, T. G. Terentyeva, A. Pakdel, D. Golberg, J. P. Hill and K. Ariga, ACS Nano, 2014, 8, 5240-5248.

7 C. Maerten, L. Jierry, P. Schaaf and F. Boulmedais, ACS Appl. Mater. Interfaces, 2017, 9, 28117-28138.

8 G. Rydzek, Q. Ji, M. Li, P. Schaaf, J. P. Hill, F. Boulmedais and K. Ariga, Nano Today, 2015, 10, 138-167.

9 N. Vogel, M. Retsch, C.-A. Fustin, A. del Campo and U. Jonas, Chem. Rev., 2015, 115, 6265-6311.

10 Y. Yue and H. Liang, J. Power Sources, 2015, 284, 435-445.

11 K.-I. Min, G. Yun, Y. Jang, K.-R. Kim, Y. H. Ko, H.-S. Jang, Y.-S. Lee, K. Kim and D.-P. Kim, Angew. Chem. Int. Ed., 2016, 55, 6925-6928.

12 Y. Liu, B. Liu and Z. Nie, Nano Today, 2015, 10, 278300.

13 B. M. Teo, L. Hosta-Rigau, M. E. Lynge and B. Städler, Nanoscale, 2014, 6, 6426-6433.

14 T.-L. Ha, J. Shin, C. W. Lim and I. S. Lee, Chem. Asian J., 2012, 7, 36-39.

15 X. Lu, A. Xie, Y. Zhang, H. Zhong, X. Xu, H. Liu and Q. Xie, Electrochimica Acta, 2017, 249, 79-88.

16 S. L. Hayward, D. M. Francis, M. J. Sis and S. Kidambi, Sci. Rep., 2015, 5, 14683.

17 N. Graf, E. Thomasson, A. Tanno, J. Voeroes and T. Zambelli, J. Phys. Chem. B, 2011, 115, 12386-12391.

18 D. Volodkin, Y. Arntz, P. Schaaf, H. Moehwald, J.-C. Voegel and V. Ball, Soft Matter, 2008, 4, 122-130.

19 K. Katagiri, R. Hamasaki, K. Ariga and J. Kikuchi, J. Am. Chem. Soc., 2002, 124, 7892-7893.

20 K. Katagiri, R. Hamasaki, K. Ariga and J. Kikuchi, Langmuir, 2002, 18, 6709-6711.
21 F. Sciortino, G. Casterou, P.-A. Eliat, M.-B. Troadec, C. Gaillard, S. Chevance, M. L. Kahn and F. Gauffre, ChemNanoMat, 2016, 2, 796-799.

22 F. Gauffre, F. Sciortino, G. Casterou, S. Chevance, WO 2017103534 A2, 2017.

23 F. Sciortino, M. Thivolle, M. Kahn, C. Gaillard, S. Chevance and F. Gauffre, Soft Matter, 2017, 13, 4393-4400.

24 N. K. Devaraj, P. H. Dinolfo, C. E. D. Chidsey and J. P. Collman, J. Am. Chem. Soc., 2006, 128, 1794-1795.

25 J. P. Collman, N. K. Devaraj, T. P. A. Eberspacher and C. E. D. Chidsey, Langmuir, 2006, 22, 2457-2464.

26 G. Rydzek, L. Jierry, A. Parat, J.-S. Thomann, J.-C. Voegel, B. Senger, J. Hemmerlé, A. Ponche, B. Frisch, P. Schaaf and F. Boulmedais, Angew. Chem. Int. Ed., 2011, 50, 4374-4377.

27 G. Rydzek, P. Polavarapu, C. Rios, J.-N. Tisserant, J.C. Voegel, B. Senger, P. Lavalle, B. Frisch, P. Schaaf, F. Boulmedais and L. Jierry, Soft Matter, 2012, 8, 10336-10343.

28 G. Rydzek, D. Toulemon, A. Garofalo, C. Leuvrey, J.-F. Dayen, D. Felder-Flesch, P. Schaaf, L. Jierry, S. BeginColin, B. P. Pichon and F. Boulmedais, Small, 2015, 11, 4638-4642.

29 G. Rydzek, T. Garnier, P. Schaaf, J.-C. Voegel, B. Senger, B. Frisch, Y. Haikel, C. Petit, G. Schlatter, L. Jierry and F. Boulmedais, Langmuir, 2013, 29, 10776-10784.

30 W. F. Paxton, J. M. Spruell and J. F. Stoddart, J. Am. Chem. Soc., 2009, 131, 6692-6694.

31 C. Nicosia, S. O. Krabbenborg, P. Chen and J. Huskens, J. Mater. Chem. B, 2013, 1, 5417-5428.

32 A. Glaria, M. L. Kahn, A. Falqui, P. Lecante, V. Collière, M. Respaud and B. Chaudret, ChemPhysChem, 2008, 9, 2035-2041.

33 S. O. Krabbenborg, C. Nicosia, P. Chen and J. Huskens, Nat. Commun., 2013, 4, 1667.

$34 \mathrm{~N}$. Shida, Y. Ishiguro, M. Atobe, T. Fuchigami and S. Inagi, ACS Macro Lett., 2012, 1, 656-659.

35 G. De Leener, F. Evoung-Evoung, A. Lascaux, J. Mertens, A. G. Porras-Gutierrez, N. Le Poul, C. 
Lagrost, D. Over, Y. R. Leroux, F. Reniers, P. Hapiot, Y. Le Mest, I. Jabin and O. Reinaud, J. Am. Chem. Soc., 2016, 138, 12841-12853.

36 T. S. Hansen, J. U. Lind, A. E. Daugaard, S. Hvilsted, T. L. Andresen and N. B. Larsen, Langmuir, 2010, 26, 16171-16177.

37 R. Kulbokaite, G. Ciuta, M. Netopilik and R. Makuska, React. Funct. Polym., 2009, 10, 771-778.

38 A. E. El Haitami, J.-S. Thomann, L. Jierry, A. Parat, J.C. Voegel, P. Schaaf, B. Senger, F. Boulmedais and B. Frisch, Langmuir, 2010, 26, 12351-12357.

39 Y. Chen, Q. Meng, M. Wu, S. Wang, P. Xu, H. Chen, Y. Li, L. Zhang, L. Wang and J. Shi, J. Am. Chem. Soc., 2014, 136, 16326-16334.

40 Z.-A. Qiao, P. Zhang, S.-H. Chai, M. Chi, G. M. Veith, N. C. Gallego, M. Kidder and S. Dai, J. Am. Chem. Soc., 2014, 136, 11260-11263.

41 J. Han, M. Wang, R. Chen, N. Han and R. Guo, Chem. Commun., 2014, 50, 8295-8298.

42 N. M. Sanchez-Ballester, G. Rydzek, A. Pakdel, A. Oruganti, K. Hasegawa, M. Mitome, D. Golberg, J. P. Hill, H. Abe and K. Ariga, J. Mater. Chem. A, 2016, 4, 9850-9857.

43 X.-Y. Yu, L. Yu and X. W. D. Lou, Adv. Energy Mater., 2016, 6, 1501333.

44 G. Kang, J. Yoo, J. Ahn and K. Kim, Nano Today, 2015, 10, 22-47.

45 L. Guo, J. A. Jackman, H.-H. Yang, P. Chen, N.-J. Cho and D.-H. Kim, Nano Today, 2015, 10, 213-239.

46 S. Shimizu, A. Murayama, T. Haruyama, T. lino, S. Mori, H. Furuta and N. Kobayashi, Chem. - Eur. J., 2015, 21, 12996-13003.

47 S. Acikgoz, G. Aktas, M. N. Inci, H. Altin and A. Sanyal, J. Phys. Chem. B, 2010, 114, 10954-10960.

48 K. Kanehira, T. Banzai, C. Ogino, N. Shimizu, Y. Kubota and S. Sonezaki, Colloids Surf. B Biointerfaces, 2008, 64, 10-15.

49 Q. Huang, W. Shen, Q. Xu, R. Tan and W. Song, Mater. Chem. Phys., 2014, 147, 550-556.

50 J.-P. Jolivet, C. Chanéac and E. Tronc, Chem. Commun. Camb. Engl., 2004, 481-487.

51 M. Baalousha, Sci. Total Environ., 2009, 407, 20932101.

52 A. Dochter, T. Garnier, E. Pardieu, N. T. T. Chau, C. Maerten, B. Senger, P. Schaaf, L. Jierry and F. Boulmedais, Langmuir, 2015, 31, 10208-10214.

53 K. Sadman, Q. Wang, S. H. Chen, D. E. Delgado and K. R. Shull, Langmuir, 2017, 33, 1834-1844.

54 F. Boulmedais, C. S. Tang, B. Keller and J. Vörös, Adv. Funct. Mater., 2006, 16, 63-70.
55 O. Guillaume-Gentil, N. Graf, F. Boulmedais, P. Schaaf, J. Vörös and T. Zambelli, Soft Matter, 2010, 6, 4246-4254.

56 N. Graf, F. Albertini, T. Petit, E. Reimhult, J. Vörös and T. Zambelli, Adv. Funct. Mater., 2011, 21, 16661672.

57 G. Ulrich and R. Ziessel, J. Org. Chem., 2004, 69, 2070-2083.

58 G. Rydzek, J.-S. Thomann, N. Ben Ameur, L. Jierry, P. Mésini, A. Ponche, C. Contal, A. E. El Haitami, J.-C. Voegel, B. Senger, P. Schaaf, B. Frisch and F. Boulmedais, Langmuir, 2010, 26, 2816-2824.

59 G. Casterou, V. Collière, P. Lecante, Y. Coppel, P.-A. Eliat, F. Gauffre and M. L. Kahn, Chem. - Eur. J., 2015, 21, 18855-18861. 